ARTICLE

\title{
Surface regulation enables high stability of single- crystal lithium-ion cathodes at high voltage
}

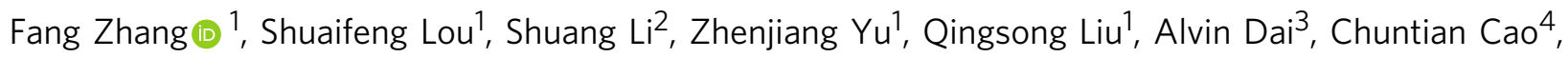
Michael F. Toney (10 ${ }^{4}$, Mingyuan Ge ${ }^{5}$, Xianghui Xiao (1) ${ }^{5}$, Wah-Keat Lee ${ }^{5}$, Yudong Yao ${ }^{6}$, Junjing Deng (1) ${ }^{6}$, Tongchao Liu ${ }^{3}$, Yiping Tang ${ }^{7}$, Geping Yin ${ }^{1}$, Jun Lu (D) ${ }^{3 凶}$, Dong Su (iD ${ }^{8 凶}$ \& Jiajun Wang ${ }^{1 凶}$

Single-crystal cathode materials for lithium-ion batteries have attracted increasing interest in providing greater capacity retention than their polycrystalline counterparts. However, after being cycled at high voltages, these single-crystal materials exhibit severe structural instability and capacity fade. Understanding how the surface structural changes determine the performance degradation over cycling is crucial, but remains elusive. Here, we investigate the correlation of the surface structure, internal strain, and capacity deterioration by using operando X-ray spectroscopy imaging and nano-tomography. We directly observe a close correlation between surface chemistry and phase distribution from homogeneity to heterogeneity, which induces heterogeneous internal strain within the particle and the resulting structural/performance degradation during cycling. We also discover that surface chemistry can significantly enhance the cyclic performance. Our modified process effectively regulates the performance fade issue of single-crystal cathode and provides new insights for improved design of high-capacity battery materials.

\footnotetext{
${ }^{1}$ MIIT Key Laboratory of Critical Materials Technology for New Energy Conversion and Storage, School of Chemistry and Chemical Engineering, Harbin Institute of Technology, 150001 Harbin, China. ${ }^{2}$ Center for Functional Nanomaterials, Brookhaven National Laboratory, Upton, NY 11973, USA. ${ }^{3}$ Chemical Sciences and Engineering Division, Argonne National Laboratory, Lemont, IL 60439, USA. ${ }^{4}$ SSRL Materials Science Division, SLAC National Accelerator Laboratory, Menlo Park, CA 94025, USA. ${ }^{5}$ National Synchrotron Light Source II, Brookhaven National Laboratory, Upton, NY 11973 , USA. ${ }^{6}$ Advanced Photon Source, Argonne National Laboratory, Lemont, IL 60439, USA. ${ }^{7}$ College of Material Science and Engineering, Zhejiang University of Technology, 310014 Hangzhou, China. ${ }^{8}$ Beijing National Laboratory for Condensed Matter Physics, Institute of Physics, Chinese Academy of Sciences, 100190 Beijing, China.

凶email: junlu@anl.gov; dongsu@iphy.ac.cn; jiajunhit@hit.edu.cn
} 
S pecific capacity improvement has been reported in high $\mathrm{Ni}$ content lithium nickel cobalt manganese oxide (NCM) electrodes for lithium-ion batteries (LIBs) charged at high voltage. However, the increased voltage cutoffs also aggravate material decomposition and impede the battery performance ${ }^{1-3}$. It is commonly accepted that these transitions from layered to spinel or rock-salt phases, and migration/segregation of transition metals (TMs) induce structural reconstruction that facilitates capacity fade ${ }^{4-6}$. During delithiation, the layered oxide material may transform into a spinel-type phase and then to a completely disordered rock salt-type structure, which is believed to inhibit the diffusion of lithium ions. In addition, the dissolution, migration, and segregation of TMs on the surface further deteriorate battery performance ${ }^{7-9}$. Although some traditional methods doping and coating strategies have been reported to inhibit cation mixing and suppress interfacial reactions, the excessive coating layer $(>20 \mathrm{~nm})$ and unregulated doping strategy may hinder the migration of $\mathrm{Li}^{+}$, resulting in poor rate capability $^{10,11}$. Therefore doping strategies would consequently decrease specific capacity. Another issue was recently raised that uneven stresses, observed in cycles driven at high voltage, induced intragranular cracks in polycrystalline, which exacerbated structural collapse and capacity loss in Ni-rich $\mathrm{NCM}^{12-14}$. To solve the deficiencies caused by different reasons, creative strategies are necessary to improve structural stability in NCM particles ${ }^{15}$. Ideal methods should be able to simultaneously tune structural and morphological characteristics to restrict both structural failure and intragranular cracks $13,16,17$.

Single crystalline NCM with only one grain for one particle (grain sizes of $2-5 \mu \mathrm{m}$ ) have attracted increasing attention for the cathode of LIBs due to their superior capacity retention during long cycle times, which exceeds conventional polycrystalline battery particles ${ }^{18,19}$. It was believed that such good capacity retention originated from their high levels of structural stability ${ }^{19-22}$. First, the cracking problems of NCM can be potentially suppressed because of the exhibited intrinsic integrity and continuous conductive networks in single-crystal particles ${ }^{23}$. Second, without grain boundaries in particles, single-crystal electrodes were theorized to provide increased oxygen loss resistance and sound structural stability in interactions with electrolyte when compared with polycrystalline materials ${ }^{24}$. Although singlecrystal NCM electrodes can eliminate the grain boundary resistance, degradations from NCM electrodes itself still occur and intrinsically hinder the further improvement of practical applications. Few studies elucidated the deterioration mechanism of single-crystalline NCM at high voltage. Understanding the structure-performance correlations of NCM single crystal can not only solve the above issue but also provide fundamental insights into the degradation mechanism of polycrystalline NCM electrodes, with a clarification on the role of the grain boundaries.

In this work, we seek to understand underlying recession mechanisms in single-crystalline battery materials with using operando synchrotron X-ray spectroscopic microscopy and X-ray nanotomography. Ni-rich single-crystal $\mathrm{LiNi}_{0.6} \mathrm{Co}_{0.2} \mathrm{Mn}_{0.2} \mathrm{O}_{2}$ (NCM622) was selected as a model electrode because it exhibits a high specific capacity of over $220 \mathrm{mAh} \mathrm{g}^{-1}$. To correlate structural/morphological changes with cycle capability, mesoscale phase distributions during long-term cycling were visualized at single-crystalline levels. Results from comprehensive testing reveal that surface physical character, such as phase transitions from homogeneity to heterogeneity during cycling, induce particle crack formations, and play a dominant role in the structural robustness of single crystals. Moreover, we discover that surface regulated approaches could mitigate this undesirable phase evolution in single-crystal NCM and significantly enhance cycle performance. Our gather evidence consequently elucidates the relationship between surface chemistry, phase transition, and performance retention, while providing new guidelines for the rational design of high performance, stable, layered cathode materials ${ }^{25-27}$.

\section{Results and discussion}

Performance decay of single crystals at high-voltage cycling. Singe-crystalline NCM particles exhibit well-defined polyhedral shapes with particle sizes of 1-5 $\mu \mathrm{m}$ (Fig. 1a and Supplementary Fig. 1). We have performed a transmission electron microscope (TEM) analysis on single particles of NCM. From the bright-field TEM images and corresponding selected area electron diffraction patterns, as shown in Supplementary Fig. 2, we concluded that these single particles are single crystal. Major X-ray diffraction (XRD) reflections in Fig. $1 \mathrm{~b}$ can be indexed as $R-3 m$ space group (JCPD, 09-0063), which is in good agreement with the hexagonal $\alpha-\mathrm{NaFeO}_{2}$ type crystal structure ${ }^{21}$. The clear splitting peaks of $(006) /(012)$ and $(018) /(110)$ imply a well-organized crystalline layered structure. Rietveld refinement exhibits a well-ordered layered structure with no evidence of other impurity phases. Also, the results show that the mixing of $\mathrm{Li} / \mathrm{Ni}$ in the NCM is common in the synthesis of layered oxides (Supplementary Table 1). NCM single crystals showed a high specific capacity of $197.8 \mathrm{mAh} \mathrm{g}^{-1}$, which is equivalent to most reported results in NCM samples (Fig. 1c). Furthermore, we carried out electrochemical stability measurement with different upper cutoff voltages $(4.1,4.3$, and $4.7 \mathrm{~V}$ ) (Fig. 1d and Supplementary Fig. 3). When cut-off voltages were below $4.3 \mathrm{~V}$, single-crystal NCM exhibited significantly increased cycling performance. However, at a high charge cutoff voltage of $4.7 \mathrm{~V}$, single-crystal NCM displayed decreased stability with a capacity retention of only $25.6 \%$ after 200 cycles. Nevertheless, in order to increase specific capacity, battery materials must be stable at higher charge voltages. Therefore, insight into the failure mechanism of high-voltage NCM single-crystal cathodes holds great value for capacity enhancement.

Structural analysis of cycled single-crystal material. The surface chemistry of single-crystal NCM was conducted with TEM, and the line scan and mapping of the electron energy loss spectroscopy reveal the presence Ni-rich phase on the surface (Fig. 2a, b and Supplementary Fig. 4), which often corresponds to a rock-salt phase $\mathrm{e}^{5,28}$. To further visualize this surface structure at atomic resolutions, we performed high-angle annular dark-field (HAADF)-scanning transmission electron microscopy (STEM) on pristine single-crystal NCM. The inner zone of NCM corresponds to the layered phase, while the surface region $(\sim 3 \mathrm{~nm})$ shows a rock-salt phase, which confirms the presence of a thin Ni-rich rock-salt layer on the single-crystal particle surface (Fig. 2c) ${ }^{29}$. As for polycrystal sample, it is generally accepted that the surface configuration of this thin rock-salt layer can significantly affect the electrochemical behavior of NCM layered oxide cathode (Fig. 2d). Nevertheless, the true relationship between surface chemistry and structural stability for single crystal NCM remains unclear, which requires comprehensive studies to understand its configuration and mechanism of impact on electrochemical performance.

Electrodes harvested from cells were analyzed using various techniques to understand the structural evolution and degradation of single-crystal NCM at high potential. First, synchrotron XRD was performed on samples cycled at a high cut-off voltage $(4.7 \mathrm{~V})$ to understand structural transformations. As shown in Fig. 3a, the shift at the (003) peak indicates low reversibility of lithium deintercalation in single-crystal NCM after 200 cycles $^{23}$. Fine chemical and structural information were also analyzed using hard X-ray absorption spectroscopy (Fig. 3b). The cycled single-crystal electrode exhibits a $\sim 1.5 \mathrm{eV}$ edge energy shift 
a

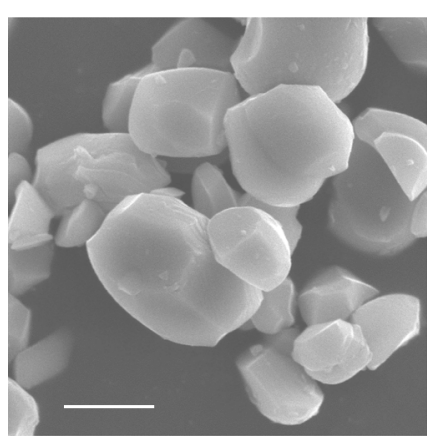

C

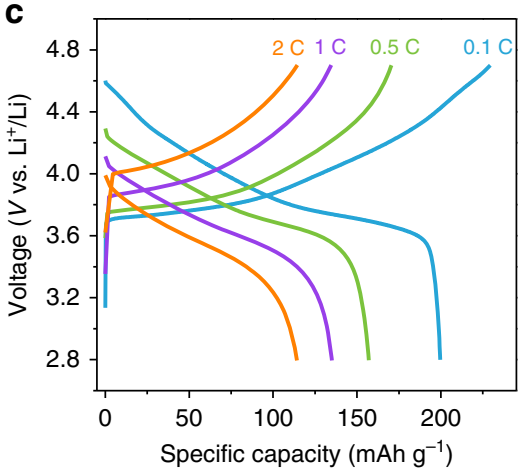

b

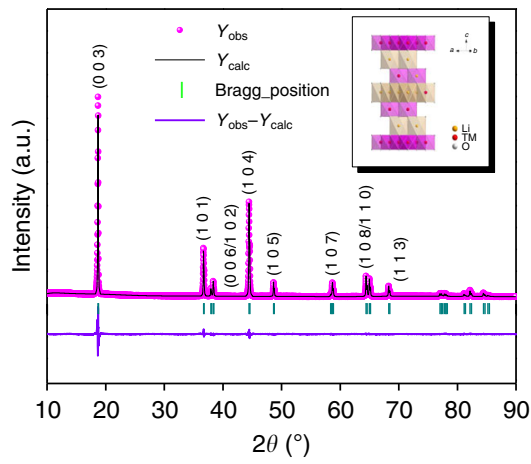

d 200

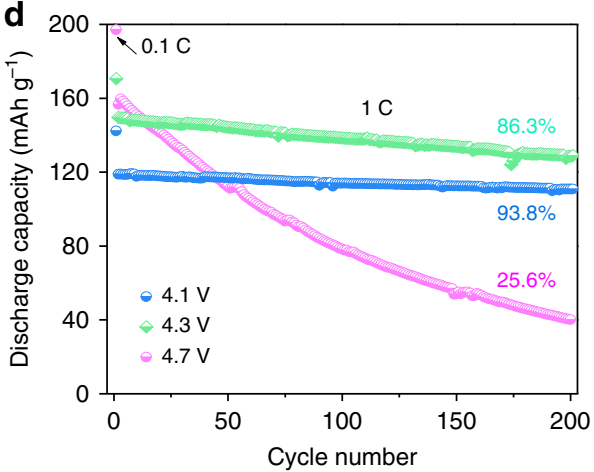

Fig. 1 Performance decay of single-crystal batteries at high-voltage cycling. a SEM images of NCM. Scale bars $2 \mu \mathrm{m}$. $\mathbf{b}$ Rietveld refinement results for NCM samples (the illustration is the crystal structure of NCM). c Charge-discharge profiles of NCM at different rates. $\mathbf{d}$ Cycling performances at $1 \mathrm{C}$ $\left(1 \mathrm{C}=270 \mathrm{~mA} \mathrm{~g}^{-1}\right)$ under different cut-off voltages $(4.1,4.3,4.7 \mathrm{~V})$.
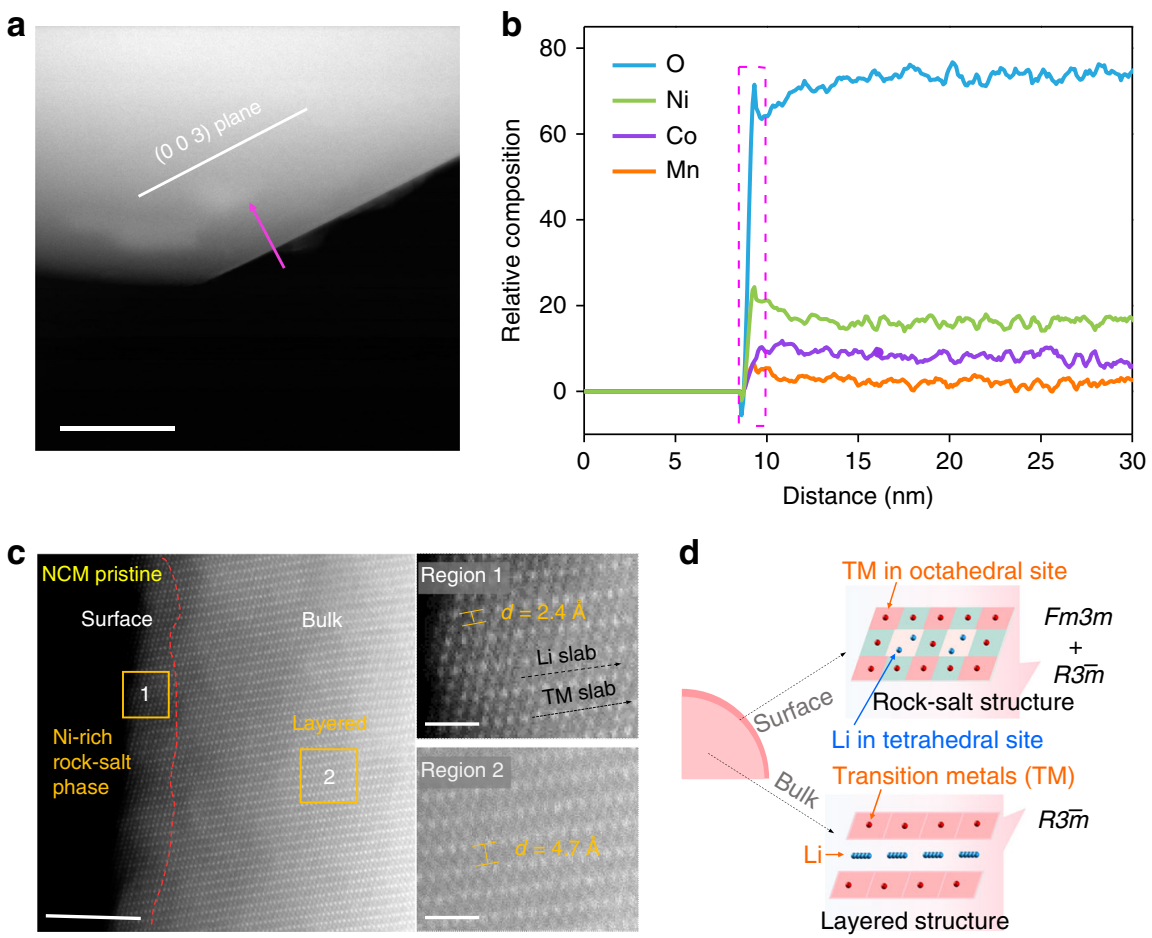

Fig. 2 Surface analysis of pristine NCM electrodes. a STEM image of NCM. b A line scan of EELS showing the elemental profiles of the relative atomic concentration of $\mathrm{Ni}, \mathrm{Co}, \mathrm{Mn}$ on pristine NCM particle surface. c Atomic resolution HAADF-STEM images of pristine NCM samples. Scale bars $5 \mathrm{~nm}$, region 1: $1 \mathrm{~nm}$, region 2: $1 \mathrm{~nm}$. d Schematic diagram for atomic configuration of NCM bulk and surface. 
a

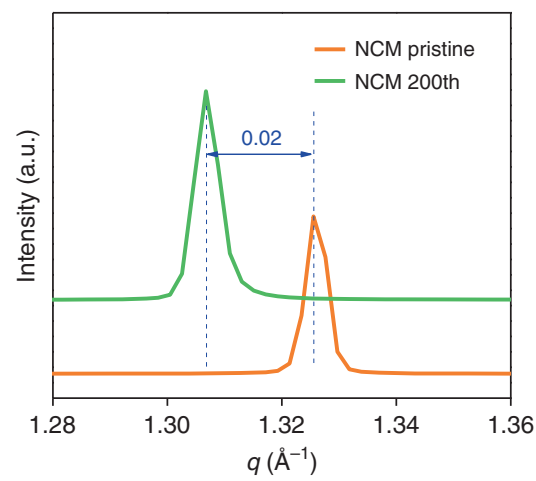

C

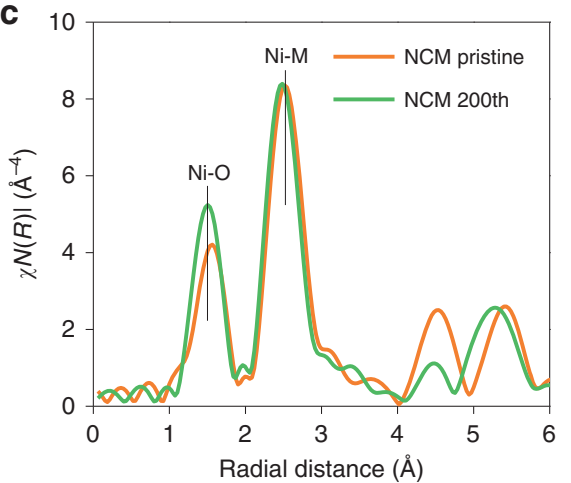

e

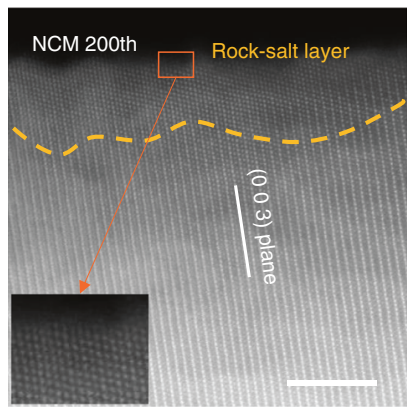

b

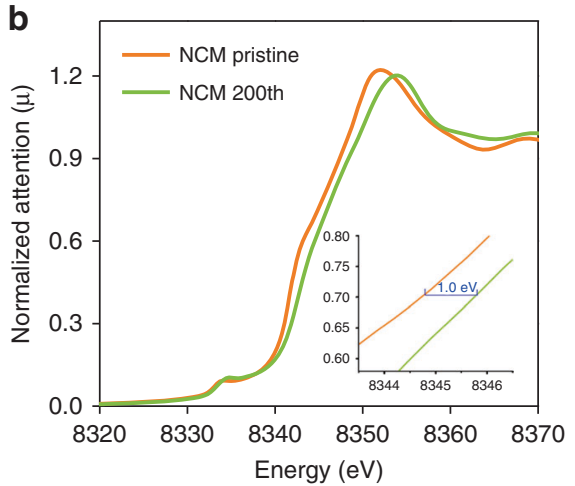

d

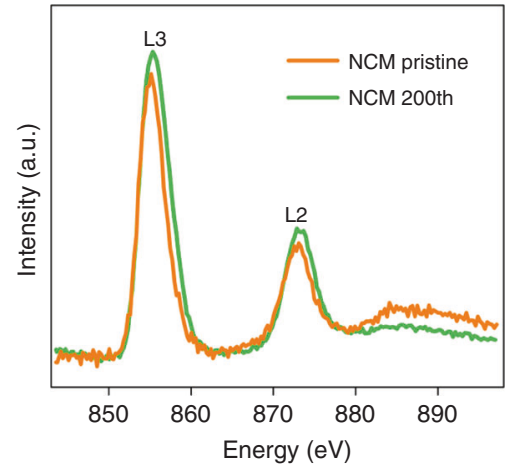

f

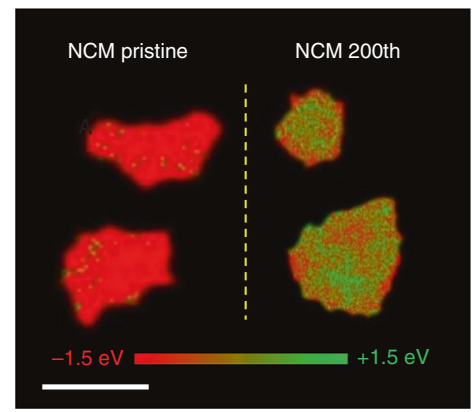

Fig. 3 Structural analysis of pristine and cycled NCM electrodes. a Synchrotron XRD pattern for NCM. b, c Ni K-edge XANES and EXAFS spectra of pristine and cycled NCM crystals. d Soft XAS spectra of Ni L-edge for pristine and 200th cycle NCM. e Atomic resolution HAADF-STEM images of 200th cycled NCM samples. $\mathbf{f}$ Ex-situ 2D TXM of pristine and cycled NCM. Scale bars $2 \mu \mathrm{m}$.

toward higher energy in the $\mathrm{Ni} \mathrm{K}$-edge $\mathrm{X}$-ray absorption near edge structure (XANES) spectrum, which reflects an oxidation state increase for $\mathrm{Ni}$ in single-crystal NCM. Extended X-ray absorption fine structure (EXAFS) spectroscopy results (Fig. 3c), which are more sensitive to local chemical and structural changes, clearly show that $\mathrm{Ni}-\mathrm{O}$ and $\mathrm{Ni}-\mathrm{M}$ interatomic distances are shortened after electrochemical cycling. Changes in the chemical state of $\mathrm{Ni}$ and the atomic environment could be ascribed to the presence of unfilled lithium vacancies during lithiation ${ }^{30}$. Surfacesensitive soft X-ray absorption spectroscopy (XAS) measurements were also conducted to further understand the surface chemical change in single-crystalline NCM. Nickel L-edge spectra have an $\mathrm{L}_{3}\left(2 p_{3 / 2} \rightarrow 3 d\right)$ and an $\mathrm{L}_{2}\left(2 p_{1 / 2} \rightarrow 3 d\right)$ region due to spin-orbital coupling. The shape, energy, position, and peak intensity ratio contain information on sample valence states. A decreased $\mathrm{L}_{3} / \mathrm{L}_{2}$ ratio was found in the spectrum after cycling, which provides evidence of $\mathrm{Ni}$ elements that have undergone oxidation reactions in at the single-crystal surface (Fig. $4 \mathrm{~d})^{31}$ This result was also confirmed with X-ray photoelectron spectroscopy analysis (Supplementary Fig. 5). With such comprehensive X-ray analysis, we decisively conclude that serious structural degradation and surface configuration transitions occur in single-crystal NCM after high-voltage cycling (Fig. 1c).

The heterogeneity of phase distribution has been observed in polycrystalline $\mathrm{NCM}^{32}$. It is generally accepted that this phase heterogeneity at the chemically delithiated samples is similar to that of electrochemically charged samples in large polycrystals. However, the mapping study of phase distribution was seldom reported in single-crystal battery materials ${ }^{33}$. Therefore, we propose that surface configuration plays a role in the cycling capability of pristine single-crystal particles. After 200 cycles, the single crystals showed a disordered layer $(\sim 5 \mathrm{~nm}$ depth) on the surface of the particle as expected (Fig. 3e), which suggests that the cycled cathode exhibited extreme instability. This surface $\mathrm{Ni}$ rich rock-salt phase will hinder lithium-ion transport, and we speculate that it will affect the inner chemical phase of the singlecrystal particles during long-term cycling. In order to further quantitatively describe structural transformation at particlescales, we conducted spatially resolved synchrotron transmission $\mathrm{X}$-ray microscopy (TXM) to probe chemical changes in single NCM crystals after cycling (Fig. 3f). Inhomogeneous distributions of $\mathrm{Ni}^{3+}$ and $\mathrm{Ni}^{4+}$ oxidation states were found to coexist in the 


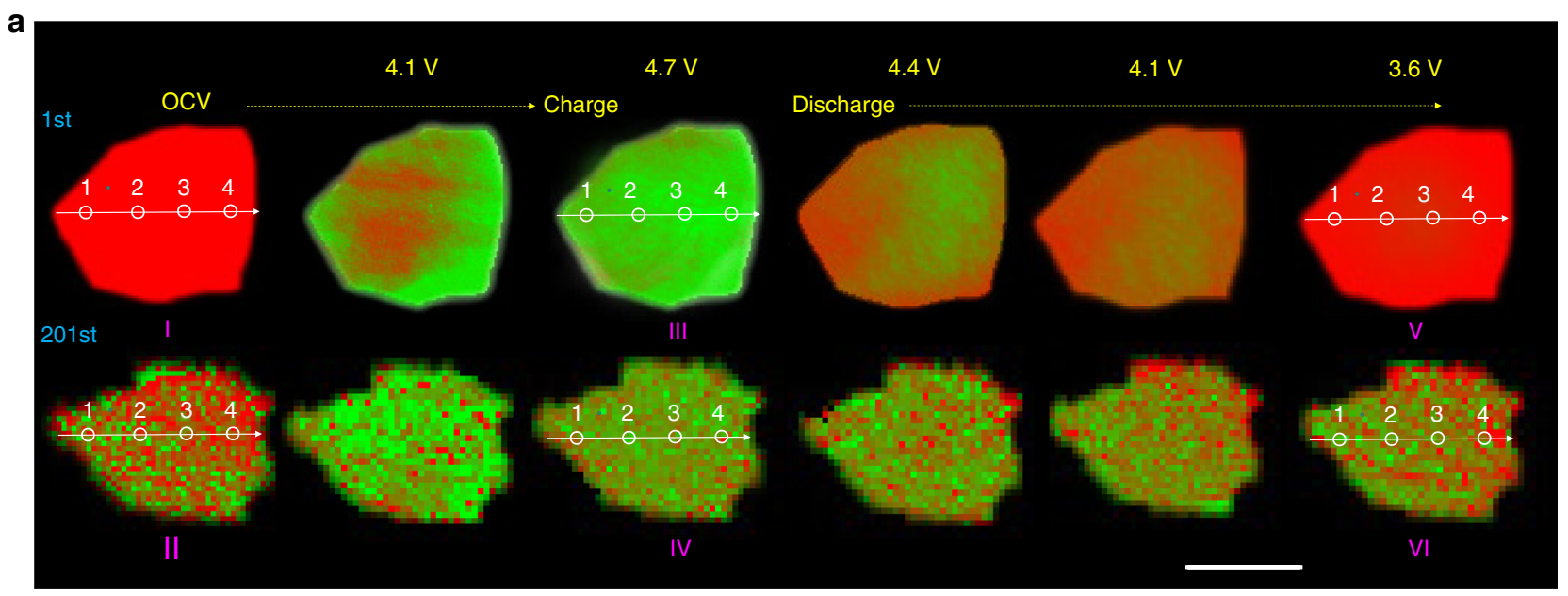

$-1.5 \mathrm{eV}$
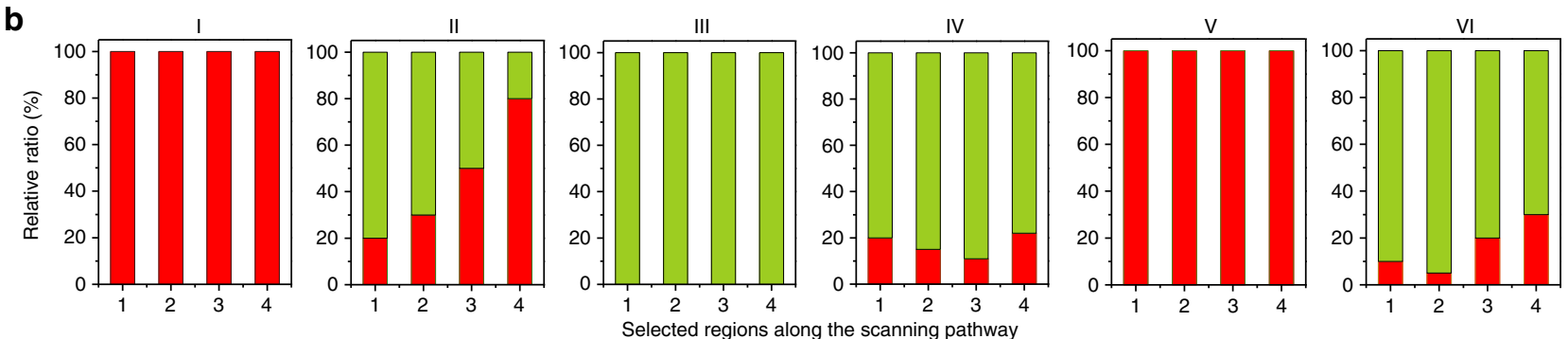

Fig. 4 Spatial-resolved electrochemical evolution after the 1st and 201st cycle. a Operando 2D chemical phase mappings at the Ni K-edge of NCM particles during the first and 201st cycles. Scale bars $2 \mu \mathrm{m}$. 1st (I, pristine; III, $4.7 \mathrm{~V} ; \mathrm{V}, 3.6 \mathrm{~V}$ ), and 201st cycle (II, pristine; IV, $4.7 \mathrm{~V}$; VI, 3.6 V), b Quantification of the selected positions in 2D TXM-XANES phase mappings (Red represents discharge state, green represents charge state).

single-crystal particle, which indicated irreversible chemical changes to NCM after battery cycling. This inhomogeneous phase distribution may be caused by lithium-ion diffusion within single crystals, which is related to surface chemical features in single-crystal particles.

Spatially-resolved structural evolution. In order to gain a deeper understanding of the correlation between surface chemistry and performance decay, in operando TXM was performed to monitor the compositional and structural evolution before and after 200 cycles ${ }^{25,34}$. During the initial cycle, the redox reaction occurs along all directions and phase-transition fronts propagate drastically, as visualized by the in operando quantitative chemical mapping in Fig. 4a. A near-complete phase transition occurs at the final charge and discharge stage, indicating highly electrochemical reversibility. Nevertheless high reversibility cannot survive after 200 cycles, which is unambiguously revealed through operando TXM chemical phase mapping. During the 201st charge cycle, it was found that around $50 \%$ of the phase composition could not recover to a pristine state, and a highly heterogeneous phase distribution appears in single-crystal NCM. Moreover, phase transition at the same location is highly irreversible during the 201st cycle, as evidenced in the composition analysis of Fig. 3b. Four representative regions from the bulk to the surface were selected and the corresponding content of $\mathrm{Ni}$ oxidation states were illustrated in Fig. 4b. The 2D histogram highlights inhomogeneous Ni oxidation state distribution within the singlecrystal particle. Reaction heterogeneity and high irreversibility could be ascribed to the characteristics of surface chemicals (Nirich rock-salt phase) on pristine single NCM crystals, which may induce heterogeneous internal strain within the particle, and further result in structural/performance degradation, as evidenced with the chemical composition analysis of Fig. $4 \mathrm{~b}$ and Supplementary Fig. 6.

To probe the influence of trace surface elemental rearrangement on chemical and physical functions, we sought a novel analysis tool with high elemental sensitivity. X-ray fluorescence microscopy (XFM) provides unparalleled sensitivity for trace element distribution measurements in many micrometer-thick specimens (true microscale battery particles) and facilitates significantly improved sensitivity relative to electron probes ${ }^{35}$. With X-ray ptychrography, an emerging method that images ultrastructures at beyond-focus-optic resolution, a combined approach with XFM and ptychography can be employed to study elemental localization within the high-resolution structural context, which aids the elucidation of phase transition mechanisms ${ }^{36-38}$. The fluorescence maps ( $\mathrm{Mn}, \mathrm{Co}, \mathrm{Ni}$ ) in Fig. 5 indicate homogeneous elemental distributions of $\mathrm{Ni}, \mathrm{Co}$, and $\mathrm{Mn}$ within the pristine single-crystalline particle, while $\mathrm{Mn}$ metal segregation and $\mathrm{Ni}$ deficiencies are observed within the single-crystalline particle after 200 cycles $^{7}$. The phase images given by ptychography reveals the projected electron density distribution of the particle along the X-ray beam direction. For the pristine sample in Fig. 5b, the phase map shows a single intact crystal with several facets, which is consistent with the SEM image of pristine particles (Fig. 1a). However, ptychographic image of the particle after 200 cycles shows inhomogeneous morphology, several example locations indicated by white arrows have lower electron density, which is presumably due to the formation of cracks. In addition, small particles with weak phase (indicated by black arrows in Fig. 5c) were observed around the particles, which come from the electrolyte. In all, the projected phase image of the cycled particle is well consistent 
a

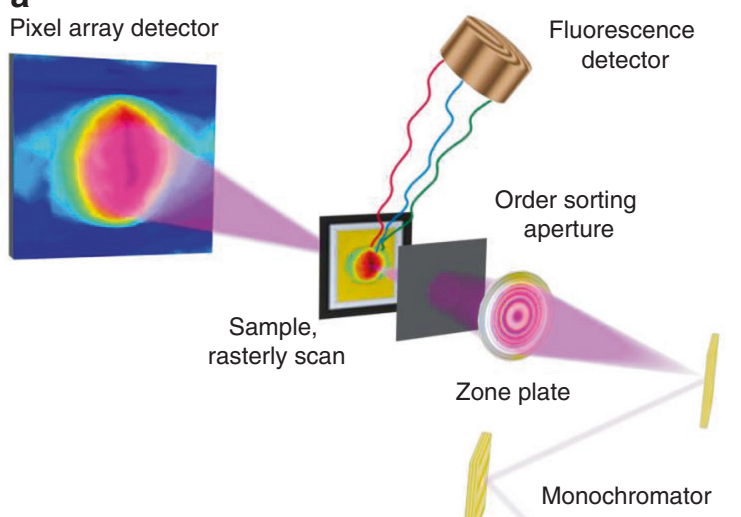

Monochromator

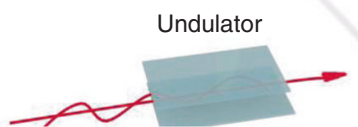

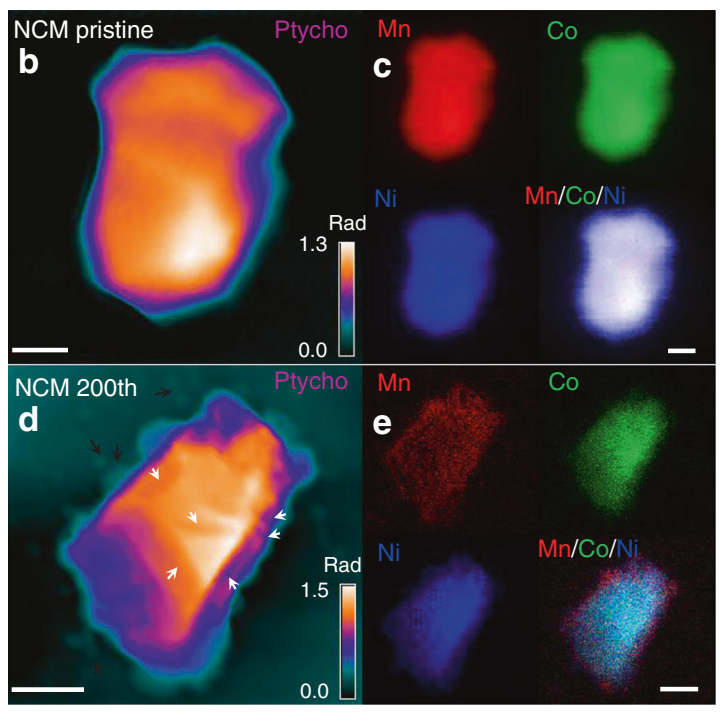

Fig. 5 Simultaneous $\mathbf{X}$-ray fluorescence and ptychography. a Experimental schematics. $\mathbf{b}$-e $\mathbf{X}$-ray fluorescence and ptychographic images of pristine and 200th cycled NCM particles to show element distribution and morphology. Scale bars: b $2 \mu \mathrm{m}, \mathbf{c} 1 \mu \mathrm{m}, \mathbf{d} 2 \mu \mathrm{m}$, e $1 \mu \mathrm{m}$.

with the surface morphology given by SEM (Fig. 5d). To better visualize the internal structures and further directly correlate microstructural changes to electrochemical cycle stability, we performed synchrotron X-ray nanotomography ${ }^{39}$. Fig. 6a illustrates the 3D microstructure of pristine and 200th cycled singlecrystalline NCM. Significant amounts of cracks in singlecrystalline particles indicate that the integrity of the original microstructural morphology has been destroyed. Close examination of NCM particles after the 200th cycle reveals multiple microcracks on the particles (indicated by the arrow in Fig. 5), which are gaps between primary particles that arose from inhomogeneous diffusion during the cycling process. Figure $6 \mathrm{~b}$ reveals the internal structural information of the split sample from the $2 \mathrm{D}$ projection images extracted along the $Z$ vertical axis. For cycled samples, present internal cracks and fractures can be attributed to heterogeneous phase transition-induced internal strains during long-term cycling. This is consistent with results obtained from SEM (Fig. 6c, d).

It is well known that the Ni-rich rock-salt phase on the surface caused by cation mixing may inhibit lithium-ion transport, which can trigger surface-phase transformations from layered to rocksalt structures and induce inhomogeneous lithium-ion distribution ${ }^{40,41}$. We employed diffusion-induced stress models to understand the Electrochem-mechanical degradation mechanism and investigate stress change in single-crystal $\mathrm{NCM}^{15}$. Fig. 6e illustrates the heterogeneous stress distribution caused by the inhomogeneous distribution of lithium-ion concentration in single-crystal NCM particles. Such inhomogeneous lithium distribution may cause mismatched strains, which leads to high-stress concentrations near the phase interface (Fig. 6f, g). As cycles proceed, the particles will be lacerated when the fracture strength is unable to sustain the strains, which quickly induces polarization and plummets cycle performance. This mechanism is schematically illustrated in Fig. 6h. As for untreated NCM single crystals, the Ni-rich rock-salt phase of a single-crystal surface inhibits near-surface lithium-ion transport, which results in heterogeneous chemical particle distribution and causes stress generation. Deep lithium extraction/intercalations and stress release further increase internal strain and the presence of intergranular cracks, which decreases the structural robustness of NCM materials.
Single-crystal NCM with modified surface chemistry. Our results reveal that the surface $\mathrm{Ni}$-rich rock-salt phase plays a major role in the cycle stability of single-crystal NCM. To address this issue, efforts should be made to eliminate surface chemical reconstruction in single crystals. It is well known that lithium could redeposit into the single crystals surface lattice via oxidation of the rock-salt $\mathrm{Ni}^{2+}$ phase to layered $\mathrm{Ni}^{3+}$, which can restore the particle surface to a well-ordered lattice structure. Inspired by this finding, we attempted to modify the surface Nirich rock-salt phase using a feasible lithium source to replenish lattice sites during re-calcination under oxygen flow and hightemperatures (denoted as $\mathrm{t}-\mathrm{NCM})^{42,43}$. From a fundamental perspective, when the NCM is calcined at high temperature with $\mathrm{O}_{2}$ flow, the lithium ions from $\mathrm{LiOH}$ could return to the lattice. Simultaneously, $\mathrm{Ni}^{2+}$ in the $\mathrm{Ni}$-rich rock-salt structure can be reoxidized to $\mathrm{Ni}^{3+}$ and restored to the layered structure (Fig. 7a). SEM images in Fig. $7 \mathrm{~b}$ help conclude that our heat-treatment process has a negligent effect on particle morphology and size. From XRD analysis, the treated single crystals exhibit the layered $R-3 \mathrm{~m}$ phase which is the same as the untreated one (Fig. $7 \mathrm{c}$ ). The intensity (003) to (104) peak ratio, which is an indicator of the degree of cation mixing in layered structures with an $R-3 \mathrm{~m}$ space group, increases from 1.56 to 1.79 , which indicates a decrease in cation mixing after treatment ${ }^{29}$. (Supplementary Table 2). It could be reasoned that this vanished Ni-rich rock-salt phase may be related to the lithium source supplements during recalcination. From synchrotron X-ray absorption analysis, no drastic variation of Ni K-edge XANES in t-NCM single-crystal samples (Supplementary Fig. 7a) is observed, which suggests the average valance state of $\mathrm{Ni}$ does not change after heat treatment. Noticeably, the amplitude of the Ni-O and Ni-TM peaks in $\mathrm{Ni} \mathrm{K}$ edge EXAFS spectra decreases significantly after treatment (Supplementary Fig. 7b) This further verifies decreased LiNi6 cation ordering in transition metal layers of modified NCM and is in good agreement with prior XRD analysis ${ }^{44}$.

Slight structural changes in the single crystals before and after treatment may be easily overlooked by some conventional characterization technologies. In addition, we assume this subtle change may probably occur near-surface region of single crystals as the treatment occurs in oxygen under high temperatures. HAADF-STEM study was carried out and it was found that 

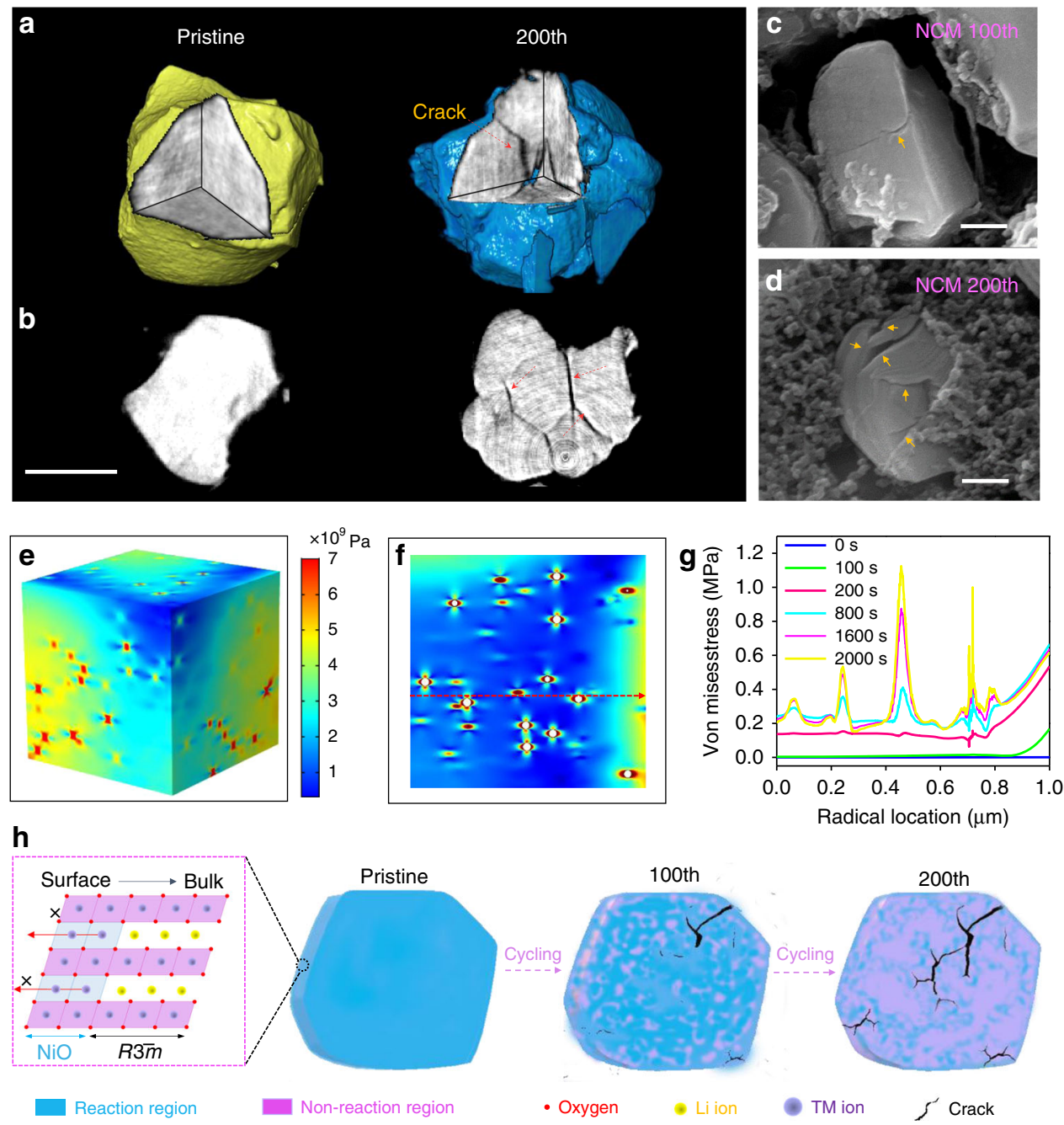

Fig. 6 3D reconstruction and structural degradation mechanism. $\mathbf{a}, \mathbf{b}$ X-ray nano-tomography reconstruction with volume rendering shows the morphological evolution of NCM after cycling. Scale bars $2 \mu \mathrm{m}$. c, d SEM images of the NCM microsphere after 100 and 200 cycles. Scale bars 500 nm. Equivalent stress within the NCM particles: e 3D semitransparent view. $\mathbf{f} 2 \mathrm{D}$ view of the cross profile. $\mathbf{g}$ Von Mises stress within the NCM particles along the imaginary line. $\mathbf{h}$ structural degradation mechanism in NCM.

t-NCM exhibits a layered phase on the surface, which indicated that our surface treatment can replenish the disordered surface structure (Fig. 7d). In the Ni L-edge spectra of the NCM and tNCM samples, the positive shift of the adsorption edge t-NCM indicates the removal of Ni-rich rock-salt structure layer after treatment (Fig. 7e $)^{31}$, which is consistent with the results obtained in Fig. 7d. We evaluated the electrochemical properties of singlecrystal t-NCM. Both initial charge/discharge capacity and electrochemical reversibility were remarkably enhanced after surface chemistry treatment (details in Supplementary Fig. 8). In particular, cycle stability is increased significantly from $25.6 \%$ to 58.8\% (Fig. 8a and Supplementary Fig. 9) and t-NCM also shows excellent rate performance (Fig. 8b). These improved electrochemical performances can be attributed to the disappearance of the Ni-rich rock-salt phase layer on the single-crystal surface. Even after 200 cycles, the layered structure of the modified singlecrystal t-NCM is still well maintained, as shown in Fig. 8c.

$\mathrm{X}$-ray technologies were further employed to understand t-NCM structural changes. Robust crystal structures with no obvious pattern change were confirmed from the XRD results of cycled 9t-NCM single crystals (Supplementary Fig. 10). Ni Kedge XANES spectra of cycled t-NCM samples display negligent edge shifts, and information on the nickel local environment
(Ni-O and Ni-TM in EXAFS) also suggest high structural stability (Supplementary Fig. 11). Soft XAS was also conducted to visualize this structural change on modified single-crystal particle surfaces and to probe surface character at the singlecrystal surface. The surface chemical states of these crystals can be determined by examining the fine structures of $\mathrm{L}_{3}, 2$ absorption edges. As revealed in Fig. 8d, the low-energy shoulder of $\mathrm{L}_{3}$-edge and the $\mathrm{L}_{3} / \mathrm{L}_{2}$ intensity ratio show negligible change in t-NCM before and after 200 cycles, which is indicative of high surface-chemical stability. In addition, t-NCM crystals retain homogeneous phase distribution across single crystals, as illustrated in X-ray spectroscopy imaging via TXM (Supplementary Fig. 12). These cumulative results highlight the crucial influence of surface chemistry on cycle capability in singlecrystal battery materials. We, therefore, suggest that homogeneous phase distribution in single crystals during delithiation contributes to a negligible lithium-ion concentration gradients, which delivers small variations in stress distribution over all particles (Fig. 8e, f) and ensures excellent cycle performance. In this work, t-NCM tailored with a feasible method retains superior surface chemistry stability, contributing to homogeneous phase transformations observed in particles during longterm electrochemical cycling. As a result, the structural integrity 
a
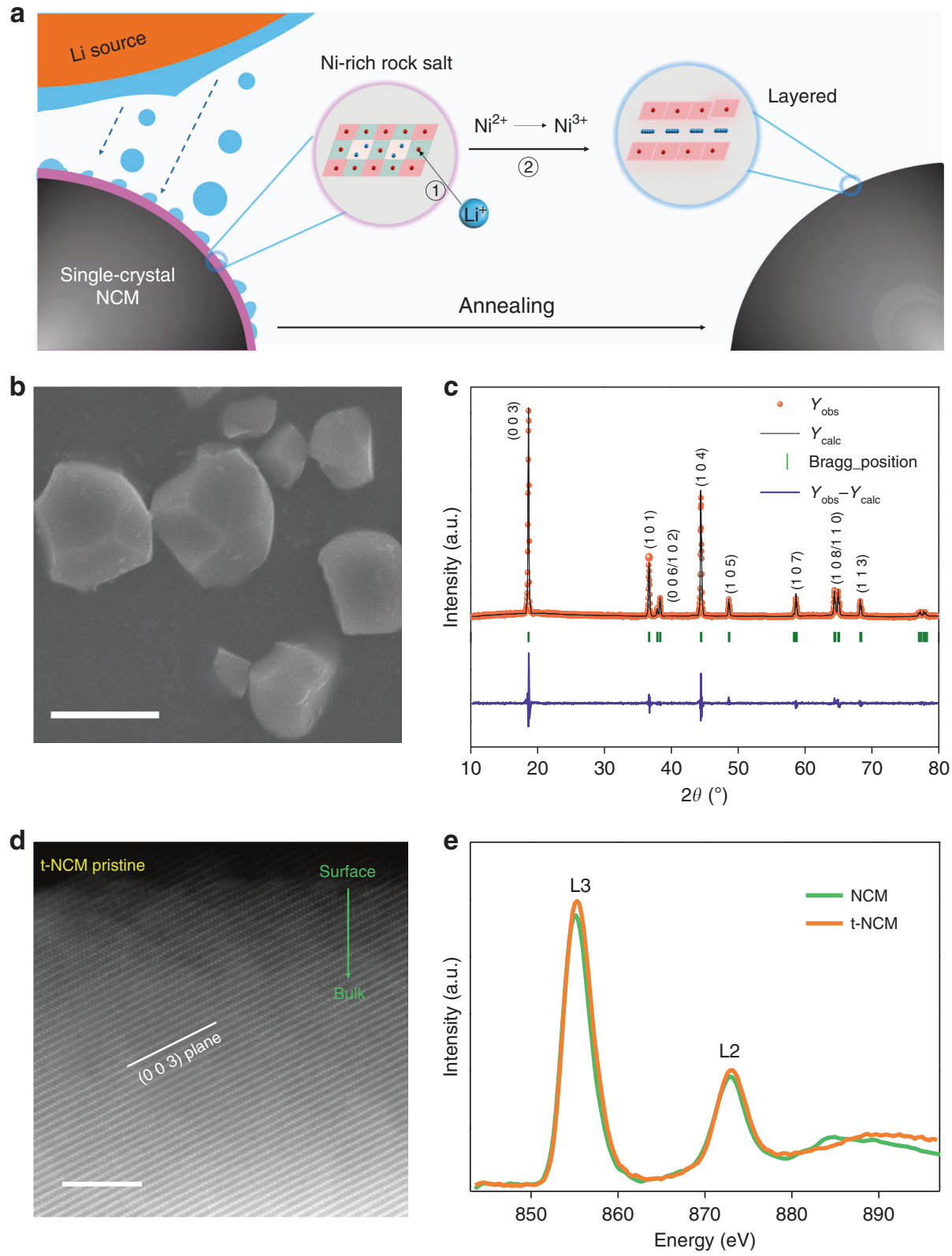

Fig. 7 Surface structure and morphology analysis of the t-NCM. a Diagram of the synthesis process and mechanism for modified NCM. $\mathbf{b}$ SEM image of pristine t-NCM. c XRD of pristine t-NCM d Atomic resolution HAADF-STEM images of pristine and cycled t-NCM samples. Scale bars $5 \mathrm{~nm}$. e Soft XAS spectra of Ni L-edge for t-NCM.

of t-NCM was well preserved and excellent electrochemical performance could be achieved.

\section{Discussion}

Understanding the fundamental role of surface chemistry in single-crystal structure stability can offer new insights into $\mathrm{Ni}$ rich, single-crystal, cathode materials used for lithium-ion batteries. In this work, the structural evolution and degradation mechanisms of Ni-rich single-crystal NCM622 were studied. Within operando X-ray imaging, spectroscopic technologies, and diffraction methods, we found that local structures around $\mathrm{Ni}$ cations were more severely disordered during long-term cycling, and unambiguously demonstrate close correlations between surface chemical character, phase transformation, and structural stability in single-crystal battery particles. Pristine surface chemistry, accompanying phase heterogeneity, and induced stresses deteriorate structural integrity along with cycle performance. Surface chemistry regulation can induce homogeneous phase distribution across single crystals, contributing to the improved surface chemical stability and performance retention. Our studies have shed new light on microstructural and chemical evolution in single-crystal particles, and offer insights into particle-level degradation mechanisms, which guides the development of advanced single-crystal battery materials with improved electrochemical and safety characteristics.

\section{Methods}

Materials synthesis. Large single-crystal NCM were synthesized using coprecipitation methods ${ }^{22,45}$. $\mathrm{Ni}_{0.6} \mathrm{Mn}_{0.2} \mathrm{Co}_{0.2}(\mathrm{OH})_{2}$ precursors: A co-precipitation method was used here to fabricate $\mathrm{Ni}_{0.6} \mathrm{Mn}_{0.2} \mathrm{Co}_{0.2}(\mathrm{OH})_{2}$ precursors. First, nickel sulfate (99.8\%, Alfa Aesar, $\left.0.6 \mathrm{~mol} \mathrm{~L}^{-1}\right)$, cobalt sulfate $\left(99.8 \%\right.$, Alfa Aesar, $\left.0.2 \mathrm{~mol} \mathrm{~L}^{-1}\right)$, and manganese sulfate $\left(99.8 \%\right.$, Alfa Aesar, $0.2 \mathrm{~mol} \mathrm{~L}^{-1}$ ) were mixed to obtain a uniform metal ion solution. Ammonia water, a complexing agent, was then used to perform a complexation reaction with the metal ions. The obtained solution was subsequently placed in a reaction kettle with $\mathrm{NaOH}$ solution, heated, and stirred to induce the coprecipitation reaction. Finally, the mixed solution was centrifuged and dried to obtain a 

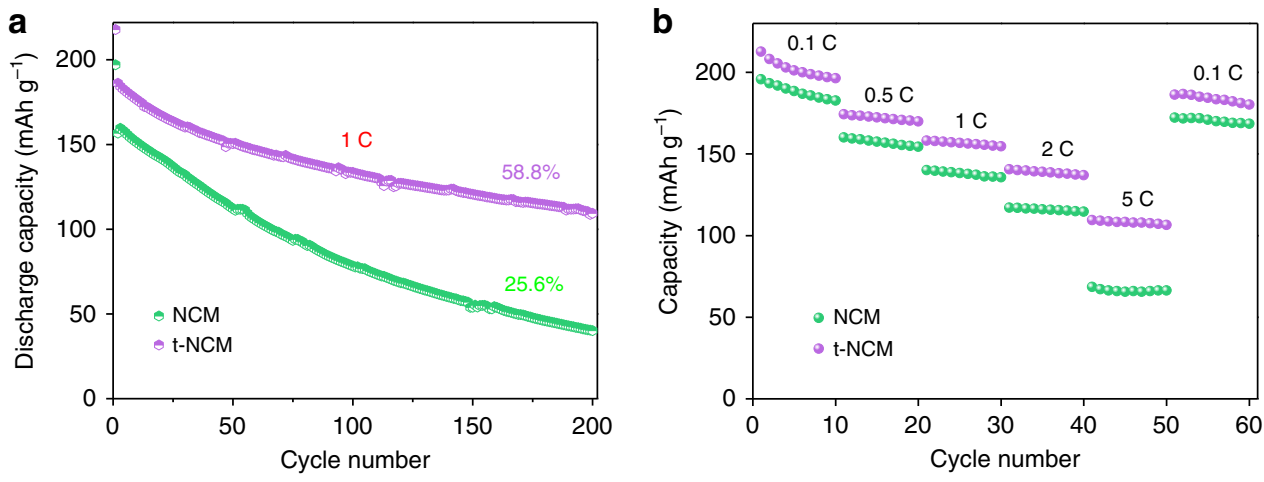

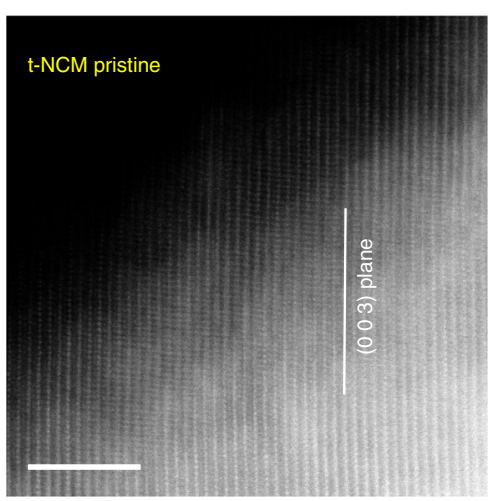

e

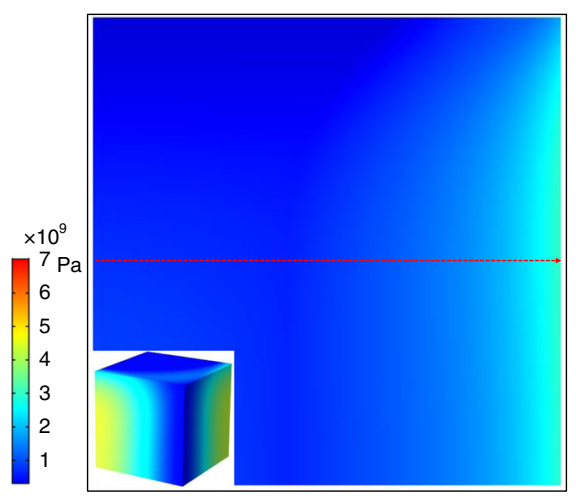

d

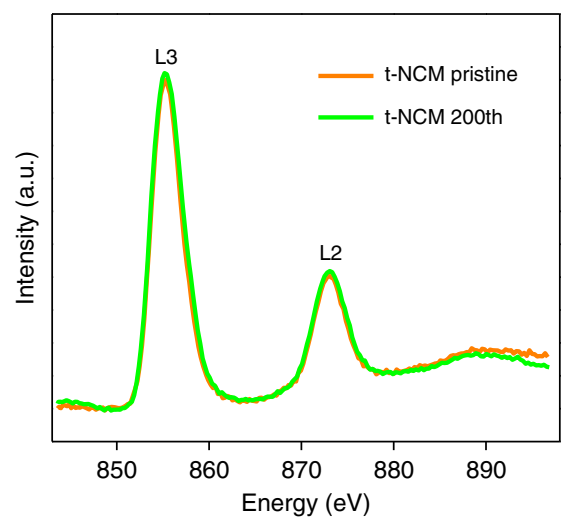

f

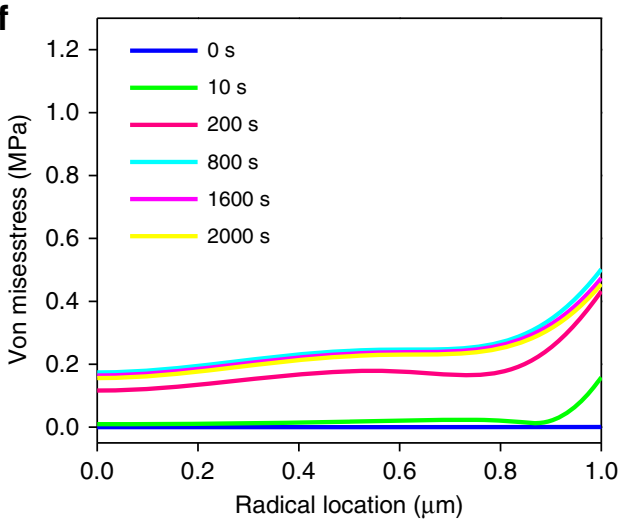

Fig. 8 Structural analysis of improved stability in the t-NCM. a Cycling performance comparison plot of untreated NCM and t-NCM. b NCM and t-NCM rate capabilities. c Atomic resolution HAADF-STEM images of pristine and cycled t-NCM samples. Scale bars $5 \mathrm{~nm}$. d Soft XAS spectra of Ni L-edge for t-NCM. Equivalent stress within the NCM particles: e 2D view of the cross profile. The illustration in the picture is 3D semitransparent view. $\mathbf{f}$ Von Mises stress within the NCM particles along the imaginary line.

nickel-cobalt-manganese precursor. Dried precursors obtained from the process steps were pre-calcined at $480^{\circ} \mathrm{C}$ for $10 \mathrm{~h}$.

NCM and $t$-NCM. single-crystal NCM materials were produced using hightemperature sintering. Mixing the NCM precursor with $\mathrm{LiOH} \cdot \mathrm{H}_{2} \mathrm{O}$ (Aladdin, $99.8 \%$, Li: $\mathrm{M}$ ratio $=1.05: 1)$, and the above mixture was then calcined at $950^{\circ} \mathrm{C}$ for $10 \mathrm{~h}$ in pure oxygen to obtain single crystal $\mathrm{NCM}^{41,42,46}$. t-NCM material was prepared by a simple surface regulation method. First, the above obtained singlecrystal NCM materials were mixed with extra $\mathrm{LiOH}$ using ball milling, and then the mixtures were calcined at $800^{\circ} \mathrm{C}$ for $2 \mathrm{~h}$ under pure oxygen flow to obtain t-NCM.

Electrode preparation and electrochemical measurement. Electrochemical tests were performed in the CR2025 coin-type cells. A slurry was prepared at room temperature by mixing of active material, conductivity agent (Super P), and binder (polyvinylidene fluoride) according to a weight ratio of 80:10:10, dissolved in $\mathrm{N}$ methyl-1,2-pyrrolidone solution for $12 \mathrm{~h}$. The slurry then was coated in a current collector (Al foil) and dried in a vacuum oven for $12 \mathrm{~h}$ at $80^{\circ} \mathrm{C}$. Active material mass loading in the electrodes was $2-3 \mathrm{mg} \mathrm{cm}^{-2}$. The coin-type cells were assembled including Li foil, composite cathode, and separator (Celgard 2025) using electrolyte $\left(1 \mathrm{~mol} \mathrm{~L}^{-1} \mathrm{LiPF}_{6}, \mathrm{EC}: \mathrm{EMC}=1: 2 \mathrm{vol} \%\right)$ with $2 \mathrm{wt} \%$ vinylene carbonate as an additive in a glovebox filled with Ar.

Cycle performance and rate capacity were tested on a battery testing system (BTS-2004, Netware) ranging from 0.1 to $5 \mathrm{C}$ between 2.8 and $4.7 \mathrm{~V}$ at room temperature $\left(25^{\circ} \mathrm{C}\right)$. Cyclic voltammetry was conducted on the $\mathrm{CHI} 660 \mathrm{E}$ electrochemical workstation at $0.1 \mathrm{mV} \mathrm{s}^{-1}$ and $2.8-4.7 \mathrm{~V}$ potential. AC impedance (EIS) was performed in the PARSTAT 2273 instrument at a $5 \mathrm{mV}$ amplitude and a frequency ranged from $0.1 \mathrm{MHz}$ to $0.01 \mathrm{~Hz}$.

$X$-ray absorption spectroscopy. Hard XAS measurements were performed on beamline $4-1$ at the SLAC National Accelerator Laboratory (SLAC). Calibration was carried out using the first inflection point of the K-edge spectrum of the element foil (e.g., Ni, $8333 \mathrm{eV}$ ) as a reference. XANES and EXAFS spectra were resolved by the Athena software package. Soft XAS experiments were performed in TEY modes at beamline BL10B of the NSRL. Beam size was $1 \mathrm{~mm}$ in diameter.

Simultaneous X-ray fluorescence and ptychography. The combination of X-ray fluorescence and ptychography gives two complementary contrast modes of the sample: the fluorescence provides the distribution of many elements while XANES provides the chemical state of a single element of interest; whereas the 
ptychographic image reconstructed from diffraction patterns shows quantitative density map (or structure image) with a spatial resolution which can be much higher than the TXM. Ptychography-XANES has been demonstrated to obtain the chemical composition mapping on Li-ion battery particles with a state-of-the-art spatial resolution reaching sub- $10 \mathrm{~nm}$.

Measurements were performed on cycled NCM single crystal particles at 2-IDD fluorescence microscope at the Advanced Photon Source (APS) in Argonne National Laboratory. An $8.8 \mathrm{keV}$ monochromatic X-ray beam was focused by a Fresnel zone plate with an outermost zone width of $70 \mathrm{~nm}$ on to NCM particles. The focused X-ray probe with a diameter of about $150 \mathrm{~nm}$ was raster-fly-scanned across the sample, for every $60 \mathrm{~nm}$ sample motion a Vortex silicon drift detector (mounted at $90^{\circ}$ to the X-ray beam direction)and a Dectris Eiger X $500 \mathrm{k}$ hybrid pixel array detector ( $1.32 \mathrm{~m}$ downstream of the sample) were simultaneously triggered for $50 \mathrm{~ms}$ to record fluorescence spectra and coherent diffraction patterns, respectively ${ }^{47}$. At the end of the scan, those diffraction patterns were input into a GPU-based code to reconstruct high-resolution structure images of particles with a pixel size of $9.7 \mathrm{~nm}$ in real space ${ }^{48}$. The elemental fluorescence maps had a pixel size equal to the scan step size of $60 \mathrm{~nm}$, however, their resolution was limited by the X-ray probe size which was about $150 \mathrm{~nm}$.

2D TXM. In operando 2D TXM-XANES experiments were performed on FXI beamline at NSLS-II. These electrodes were composed of active materials, carbon black and binder (4:4:2 in weight). In the construction of cell models, carbon papers and binders are necessary, but X-rays can transmit through these substances, so the authenticity of data will not be affected. To capture the correlation of phase change to the state of charge, the phase distribution of the battery in the 1 st and 201st cycles were collected. The TXM at FXI beamline can obtain individual 2D projection images at $30 \mathrm{~nm}$ spatial resolution. A CCD camera with a field of view of $40 \times 40 \mu \mathrm{m}^{2}$ was employed, which means multiple particles can be observed simultaneously. The exposure times can be $<50 \mathrm{~ms}$ per image, so one can catch the fast electrochemical reaction process. The 2D TXM-XANES images were collected at different states of charge by scanning $\mathrm{Ni}$ element K-edge ranging from 8313 to $8413 \mathrm{eV}$, with $1 \mathrm{eV}$ step size, which generated $2 \mathrm{k} \times 2 \mathrm{k}$ XANES spectra. In this work, each image was collected with $0.02 \mathrm{~s}$ exposure time ${ }^{49}$.

HAADF-STEM. Electron tomography and HAADF-STEM imagings were collected by a JEOL JEM-2100F operated at $200 \mathrm{kV}$. HAADF-STEM images and elemental mapping analysis were conducted on a Hitachi HD2700C. And these images were captured in sufficiently thin domains of the particles, owing to the resolution is limited by the thickness of the material.

Computational method. Interfacial models were constructed for randomly distributed, non-reactive regions, that contained homogeneous phase transformations within particles. Moreover, in the simulation of stress distribution the anisotropic deformation of NCM was considered, and the detailed tensor $D_{i j}$ represents the diffusion coefficient. We set $D_{11}=D_{22}=1 \times 10^{-13} \mathrm{~m}^{2} \mathrm{~s}^{-1}, D_{33}=1 \times 10^{-18} \mathrm{~m}^{2} \mathrm{~s}^{-1}$, and $D_{i j}=0$ for the other entries. The detailed calculation process is as follows. Chemical strain invoked by lithium extraction was assumed to be proportional to the normalized lithium concentration (c) at the fully lithiated state, as $\varepsilon_{i j}^{c}=\beta_{i j} c$. The diagonal tensor, $\beta_{i j}$, represents the lithiation expansion coefficients. As for NCM622, we set $\beta_{11}=\beta_{22}=2.8 \%, \beta_{33}=-4.0 \%$, and $\beta_{i j}=0$ for the other entries ${ }^{50}$. Since it is an orthotropic crystal, the stiffness tensor of the layered structure depends on nine independent material constants. We set the material constants of $\operatorname{NCM}(c=0)$ and LiNCM $(c=1)$ in the model, and assumed that the stiffness tensor of the intermediate stages scale linearly with lithium concentration ${ }^{44}$.

Diffusion induced stress calculations were performed using the finite element method with a commercial COMSOL Multiphysics package ${ }^{41}$. Diffusion induced stress caused by the insertion and extraction of $\mathrm{Li}^{+}$was formulated following the thermal analogy. Constitutive equations describing the stress and strain are given by

$$
\begin{gathered}
\varepsilon_{i j}=\varepsilon_{i j}^{e}+\varepsilon_{i j}^{c}=\frac{1}{E}\left[(1+v) \sigma_{i j}-v \sigma_{k k} \delta_{i j}\right]+\beta_{i j} c, \\
\sigma_{r}=\lambda e+2 \mu \varepsilon_{r}-\frac{1}{3}(3 \lambda+2 \mu) \Omega\left(C-C_{0}\right), \\
\sigma_{\theta}=\lambda e+2 \mu \varepsilon_{\theta}-\frac{1}{3}(3 \lambda+2 \mu) \Omega\left(C-C_{0}\right),
\end{gathered}
$$

where $\varepsilon_{i j}$ and $\sigma_{i j}$ are the stretch tensor and stress tensor, respectively. $v$ is Poisson's ratio; $E$ is Young's modulus. For the diffusion problem, the transport of Lithium ions can be described by modified Fick's law, including the effect of stresses on diffusion:

$$
\begin{gathered}
\frac{\partial c}{\partial t}+\nabla \cdot\left(-D_{i j} \nabla c+\frac{D_{i j} c}{R T c_{r e f}} \nabla \sum \beta_{i j} \sigma_{i j}\right)=0, \\
\mu_{s}=\mu_{0}+R T \ln C-\Omega \sigma_{m},
\end{gathered}
$$

where $D_{i j}$ is the diffusion coefficient tensor, and $R$ is the universal gas constant. $T$ is the temperature.

\section{Data availability}

The data supporting the findings of this study are available within the article and its Supplementary information files, or from the corresponding authors on reasonable request.

Received: 11 March 2020; Accepted: 26 May 2020;

Published online: 16 June 2020

\section{References}

1. Goodenough, J. B. \& Park, K. S. The Li-ion rechargeable battery: a perspective. J. Am. Chem. Soc. 135, 1167-1176 (2013).

2. Yang, H. et al. Simultaneously dual modification of ni-rich layered oxide cathode for high-energy lithium-ion batteries. Adv. Funct. Mater. 29, 1808825 (2019).

3. Lee, J.-I., Song, G., Cho, S., Han, D.-Y., Park, S. Lithium metal interface modification for high-energy batteries: approaches and characterization. Battery Supercap. https://doi.org/10.1002/batt.202000016.

4. Leanza, D., Mirolo, M., Vaz, C. A. F., Novák, P. \& El Kazzi, M. Surface degradation and chemical electrolyte oxidation induced by the oxygen released from layered oxide cathodes in Li-Ion batteries. Battery Supercap. 2, 482-492 (2019).

5. Xiao, P., Shi, T., Huang, W. \& Ceder, G. Understanding surface densified phases in Ni-rich layered compounds. ACS Energy Lett. 4, 811-818 (2019).

6. Li, W. et al. Dynamic behaviour of interphases and its implication on highenergy-density cathode materials in lithium-ion batteries. Nat. Commun. 8, 14589 (2017).

7. Lin, F. et al. Metal segregation in hierarchically structured cathode materials for high-energy lithium batteries. Nat. Energy 1, 15004 (2016).

8. Or, T., Gourley, S. W. D., Kaliyappan, K., Yu, A. \& Chen, Z. Recycling of mixed cathode lithium-ion batteries for electric vehicles: Current status and future outlook. Carbon Energy 2, 6-43 (2020).

9. Lin, F. et al. Phase evolution for conversion reaction electrodes in lithium-ion batteries. Nat. Commun. 5, 3358 (2014).

10. Liu, P. et al. Lithium deficiencies engineering in Li-Rich layered oxide $\mathrm{Li}_{1.098} \mathrm{Mn}_{0.533} \mathrm{Ni}_{0.113} \mathrm{Co}_{0.138} \mathrm{O}_{2}$ for high-stability cathode. J. Am. Chem. Soc. 141, 10876-10882 (2019)

11. Shin, W., Lu, J. \& Ji, X. ZnS coating of cathode facilitates lean-electrolyte Li-S batteries. Carbon Energy 1, 165-172 (2019).

12. Becker, D. et al. Surface modification of Ni-Rich $\mathrm{LiNi}_{0.8} \mathrm{Co}_{0.1} \mathrm{Mn}_{0.1} \mathrm{O}_{2}$ cathode material by tungsten oxide coating for improved electrochemical performance in lithium-ion batteries. ACS Appl. Mater. Interfaces 11, 18404-18414 (2019).

13. Yan, P. et al. Intragranular cracking as a critical barrier for high-voltage usage of layer-structured cathode for lithium-ion batteries. Nat. Commun. 8, 14101 (2017).

14. Li, W., Asl, H. Y., Xie, Q. \& Manthiram, A. Collapse of $\mathrm{LiNi}_{1-\mathrm{x}-\mathrm{y}} \mathrm{Co}_{\mathrm{x}} \mathrm{Mn}_{\mathrm{y}} \mathrm{O}_{2}$ lattice at deep charge irrespective of nickel content in lithium-ion batteries. $J$. Am. Chem. Soc. 141, 5097-5101 (2019).

15. Zhang, H. et al. Rock-salt growth-induced (003) cracking in a layered positive electrode for Li-ion batteries. ACS Energy Lett. 2, 2607-2615 (2017).

16. Liu, H. et al. Intergranular cracking as a major cause of long-term capacity fading of layered cathodes. Nano Lett. 17, 3452-3457 (2017).

17. Zhang, H. et al. Facet-d extending the service in-situ surface modification ependent rock-salt reconstruction on the surface of layered oxide cathodes. Chem. Mater. 30, 692-699 (2018).

18. Kimijima, T. et al. Molybdate flux growth of idiomorphic $\mathrm{Li}\left(\mathrm{Ni}_{1 / 3} \mathrm{Co}_{1 / 3} \mathrm{Mn}_{1 / 3}\right)$ $\mathrm{O}_{2}$ single crystals and characterization of their capabilities as cathode materials for lithium-ion batteries. J. Mater. Chem. A 4, 7289-7296 (2016).

19. Zhu, J. \& Chen, G. Single-crystal based studies for correlating the properties and high-voltage performance of $\mathrm{Li}\left[\mathrm{Ni}_{\mathrm{x}} \mathrm{Mn}_{\mathrm{y}} \mathrm{Co}_{1-\mathrm{x}-\mathrm{y}}\right] \mathrm{O}_{2}$ cathodes. J. Mater. Chem. A 7, 5463-5474 (2019).

20. Kuppan, S., Xu, Y., Liu, Y. \& Chen, G. Phase transformation mechanism in lithium manganese nickel oxide revealed by single-crystal hard X-ray microscopy. Nat. Commun. 8, 14309 (2017).

21. $\mathrm{Xu}, \mathrm{X}$. et al. Radially oriented single-crystal primary nanosheets enable ultrahigh rate and cycling properties of $\mathrm{LiNi}_{0.8} \mathrm{Co}_{0.1} \mathrm{Mn}_{0.1} \mathrm{O}_{2}$ cathode material for lithium-ion batteries. Adv. Energy Mater. 9, 1803963 (2019).

22. Li, H., Li, J., Ma, X. \& Dahn, J. R. Synthesis of single crystal $\mathrm{LiNi}_{0.6} \mathrm{Mn}_{0.2} \mathrm{Co}_{0.2} \mathrm{O}_{2}$ with enhanced electrochemical performance for lithiumion batteries. J. Electrochem. Soc. 165, A1038-A1045 (2018).

23. Mohanty, D., Li, J., Nagpure, S. C., Wood, D. L. \& Daniel, C. Understanding the structure and structural degradation mechanisms in high-voltage, lithiummanganese-rich lithium-ion battery cathode oxides: a review of materials diagnostics. MRS Energy Sustain. 2, E15 (2015).

24. Yoon, W. S. et al. In situ soft XAS study on nickel-based layered cathode material at elevated temperatures: a novel approach to study thermal stability. Sci. Rep. 4, 6827 (2014). 
25. $\mathrm{Xu}, \mathrm{Y}$. et al. In situ visualization of state-of-charge heterogeneity within a $\mathrm{LiCoO}_{2}$ particle that evolves upon cycling at different rates. ACS Energy Lett. 2, 1240-1245 (2017).

26. Lee, S. Y. et al. Revisiting primary particles in layered lithium transition-metal oxides and their impact on structural degradation. Adv. Sci. 6, 1800843 (2019).

27. Mao, Y. et al. High-voltage charging-induced strain, heterogeneity, and microcracks in secondary particles of a nickel-rich layered cathode material. Adv. Funct. Mater. 29, 1900247 (2019).

28. Lin, F. et al. Surface reconstruction and chemical evolution of stoichiometric layered cathode materials for lithium-ion batteries. Nat. Commun. 5, 3529 (2014).

29. Cho, Y., Oh, P. \& Cho, J. A new type of protective surface layer for highcapacity Ni-based cathode materials: nanoscaled surface pillaring layer. Nano Lett. 13, 1145-1152 (2013).

30. Jiao, S. et al. Stable cycling of high-voltage lithium metal batteries in ether electrolytes. Nat. Energy 3, 739-746 (2018).

31. Taylor, Z. N. et al. Stabilization of O-O bonds by $\mathrm{d}^{0}$ Cations in $\mathrm{Li}^{4+} \mathrm{xNi}_{1-}$ ${ }_{\mathrm{x}} \mathrm{WO}_{6}(0 \leq \mathrm{x} \leq 0.25)$ rock salt oxides as the origin of large voltage hysteresis. $J$. Am. Chem. Soc. 141, 7333-7346 (2019).

32. Tian, C. et al. Charge heterogeneity and surface chemistry in polycrystalline cathode materials. Joule 2, 464-477 (2018).

33. $\mathrm{Xu}, \mathrm{Z}$. et al. Charge distribution guided by grain crystallographic orientations in polycrystalline battery materials. Nat. Commun. 11, 83 (2020).

34. Bak, S.-M., Shadike, Z., Lin, R., Yu, X. \& Yang, X.-Q. In situ/operando synchrotron-based X-ray techniques for lithium-ion battery research. NPG Asia Mater. 10, 563-580 (2018).

35. Wang, M. et al. Influence of Fe substitution into $\mathrm{LaCoO}_{3}$ electrocatalysts on oxygen-reduction activity. ACS Appl. Mater. Interfaces 11, 5682-5686 (2019).

36. Deng, J. et al. Simultaneous cryo X-ray ptychographic and fluorescence microscopy of green algae. Proc. Natl Acad. Sci. USA 112, 2314-2319 (2015).

37. Yang, Y. et al. Quantification of heterogeneous degradation in Li-ion batteries. Adv. Energy Mater. 9, 1900674 (2019).

38. Xu, Z., Rahman, M. M., Mu, L., Liu, Y. \& Lin, F. Chemomechanical behaviors of layered cathode materials in alkali metal ion batteries. J. Mater. Chem. A. 6, 21859-21884 (2018)

39. Xia, S. et al. Chemomechanical interplay of layered cathode materials undergoing fast charging in lithium batteries. Nano Energy 53, 753-762 (2018).

40. Kondrakov, A. O. et al. Charge-transfer-induced lattice collapse in Ni-Rich NCM cathode materials during delithiation. J. Phys. Chem. C 121, 24381-24388 (2017).

41. De Biasi, L. et al. Chemical, structural, and electronic aspects of formation and degradation behavior on different length scales of Ni-rich NCM and Li-rich HE-NCM cathode materials in Li-ion batteries. Adv. Mater. 31, 1900985 (2019).

42. Ren, Z. et al. Constant dripping wears away a stone: fatigue damage causing particles' cracking. J. Power Sources 416, 104-110 (2019).

43. Ge, M. et al. One-minute nano-tomography using hard X-ray full-field transmission microscope. Appl. Phys. Lett. 113, 083109 (2018).

44. Kim, T. et al. Operando X-ray absorption spectroscopy study of atomic phase reversibility with wavelet transform in the lithium-rich manganese based oxide cathode. Chem. Mater. 28, 4191-4203 (2016).

45. $\mathrm{Li}$, J. et al. Synthesis of single crystal $\mathrm{LiNi}_{0.5} \mathrm{Mn}_{0.3} \mathrm{Co}_{0.2} \mathrm{O}_{2}$ for lithium ion batteries. J. Electron. Mater. 164, A3529-A3537 (2017).

46. Huang, B. et al. A simple method for the complete performance recovery of degraded Ni-rich $\mathrm{LiNi}_{0.70} \mathrm{Co}_{0.15} \mathrm{Mn}_{0.15} \mathrm{O}_{2}$ cathode via surface reconstruction. ACS Appl. Mater. Interfaces 11, 14076-14084 (2019).

47. Deng, J. et al. Continuous motion scan ptychography: characterization for increased speed in coherent x-ray imaging. Opt. Express 23, 5438-5451 (2015).

48. Nashed, Y. S. et al. Parallel ptychographic reconstruction. Opt. Express 22, 32082-32097 (2014)

49. Yoon, W.-S., Chung, K. Y., McBreen, J. \& Yang, X.-Q. A comparative study on structural changes of $\mathrm{LiCo}_{1 / 3} \mathrm{Ni}_{1 / 3} \mathrm{Mn}_{1 / 3} \mathrm{O}_{2}$ and $\mathrm{LiNi}_{0.8} \mathrm{Co}_{0.15} \mathrm{Al}_{0.05} \mathrm{O}_{2}$ during first charge using in situ XRD. Electrochem. Commun. 8, 1257-1262 (2006).

50. Sun, H. \& Zhao, K. Electronic structure and comparative properties of $\mathrm{LiNi}_{\mathrm{x}} \mathrm{Mn}_{\mathrm{y}} \mathrm{Co}_{\mathrm{z}} \mathrm{O}_{2}$ cathode materials. J. Phys. Chem. C 121, 6002-6010 (2017).

\section{Acknowledgements}

This work was supported by the start-up fund and "Young Scientist Studio" of Harbin Institute of Technology, Natural Science Funds of Heilongjiang Province (No. ZD2019B001), National Natural Science Foundation of China (No. U1932205), HIT Research Institute (Zhao Yuan) of New Materials and Intelligent Equipment Technology Co., Ltd. Scientific and Technological Cooperation and Development Fund (No.2017KJHZ002). This work used resources at FXI beamline (18-ID) of the National Synchrotron Light Source-II, a U.S Department of Energy (DOE) Office of Science User Facility operated for the DOE Office of Science by Brookhaven National Laboratory under Contract No. DE-SC0012704. The electron microscopy work is supported by the Center for Functional Nanomaterials, which is a US DOE Office of Science Facility, at Brookhaven National Laboratory under Contract No DE- SC0012704. Use of the Stanford Synchrotron Radiation Lightsource, SLAC National Accelerator Laboratory, is supported by the U.S. Department of Energy, Office of Science, Office of Basic Energy Sciences under Contract No. DE-AC02-76SF00515. The work at Argonne National Laboratory was supported by the U. S. Department of Energy (DOE), Office of Energy Efficiency and Renewable Energy, Vehicle Technologies Office. Argonne National Laboratory is operated for DOE Office of Science by UChicago Argonne, LLC, under contract number DE-AC02-06CH11357. The authors gratefully acknowledge the provision of sample and constructive discussion from Prof. Pengiian Zuo in Harbin Institute of Technology.

\section{Author contributions}

F.Z. and J.W. conceived the idea and designed the experiments. F. Z., Z.Y., and Q.L. carried out the electrochemical measurements and analysis. G.Y., S.Lou., and Y.T. gave help in synthesis of single-crystal NCM and experiments. S.Li. and D.S. conducted TEM measurements and processed the TEM data. A.D., T.L., and J.L participated in data analysis and discussion. C.Cao, M.F.T. carried out the ex-situ XRD and XAS. M.G., X.X., and W.L. performed the synchrotron X-ray tomography experiments and data analysis Y.Yao and J.D. performed ex-situ synchrotron-XRF, respectively. F.Z. and J.W. wrote the manuscript and all authors edited the manuscript.

\section{Competing interests}

The authors declare no competing interests.

\section{Additional information}

Supplementary information is available for this paper at https://doi.org/10.1038/s41467020-16824-2.

Correspondence and requests for materials should be addressed to J.L., D.S. or J.W.

Peer review information Nature Communications thanks the anonymous reviewer(s) for their contribution to the peer review of this work.

Reprints and permission information is available at http://www.nature.com/reprints

Publisher's note Springer Nature remains neutral with regard to jurisdictional claims in published maps and institutional affiliations.

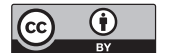

Open Access This article is licensed under a Creative Commons Attribution 4.0 International License, which permits use, sharing, adaptation, distribution and reproduction in any medium or format, as long as you give appropriate credit to the original author(s) and the source, provide a link to the Creative Commons license, and indicate if changes were made. The images or other third party material in this article are included in the article's Creative Commons license, unless indicated otherwise in a credit line to the material. If material is not included in the article's Creative Commons license and your intended use is not permitted by statutory regulation or exceeds the permitted use, you will need to obtain permission directly from the copyright holder. To view a copy of this license, visit http://creativecommons.org/ licenses/by/4.0/.

This is a U.S. government work and not under copyright protection in the U.S.; foreign copyright protection may apply 2020 